\title{
Impact of Home-to-Centre Distance on Bone Marrow Transplantation Outcomes
}

Malak Al Naabi, ${ }^{1}$ "Murtadha Al Khabori, ${ }^{2}$ Mohammed Al-Huneini, ${ }^{2}$ Abdulhakeem Al-Rawas, ${ }^{3}$ David Dennison ${ }^{4}$

$$
\text { تأثير بعد المنزل عن مركز العلاج على نتائج زراعة نخاع العظم }
$$

ملاك الناعبية، مرتضى الخابوري، محمد الحنيني، عبدالحكيم الرواس، ديفيد دينسون

ABSTRACT: Objectives: Haematopoietic stem cell transplantation (HSCT) in Oman started in 1994 at Sultan Qaboos University Hospital (SQUH), Muscat, Oman. Previous studies have suggested that longer driving time to the transplant centre (DTC) independently correlates with worse overall survival (OS). Therefore, this study aimed to examine the impact of DTC on OS and acute graft-versus-host disease (aGvHD). Methods: This retrospective study included all patients who underwent HSCT between February 2006 and December 2016 at SQUH. The DTC was determined using Google Maps (Google LLC., Mountain View, California, USA). The probability of OS was estimated using a Kaplan-Meier estimator and the impact of DTC on OS was compared using a Cox model. Results: A total of 170 patients were included in this study of which $52 \%$ were male and $28 \%$ were from the Al Batinah region. The mean age was $14.2 \pm 12.2$ years. The mean haemoglobin, platelet and white blood cell counts before the HSCT were $10.3 \pm 1.7 \mathrm{~g} / \mathrm{dL}$, $207 \pm 131 \times 10^{9} / \mathrm{L}$ and $5.1 \pm 5.9 \times 10^{9} / \mathrm{L}$, respectively. The median DTC for those with aGvHD was 84 minutes, which is similar to patients without aGvHD $(P=0.918)$. The hazard ratio for DTC as a predictor of OS was $1.0(P=0.901)$. Conclusion: In this single centre study, DTC did not impact aGvHD or OS in patients post-HSCT. The study was limited by its retrospective design and the small sample size. It is recommended that these results be confirmed in a prospective study.

Keywords: Hematopoietic Stem Cell Transplantation; Graft Versus Host Disease; Survival Analysis; Travel; Oman.

الملخص: الهدف: بدأت زراعة الخلايا الجذعية المكونة للدم في عمان عام

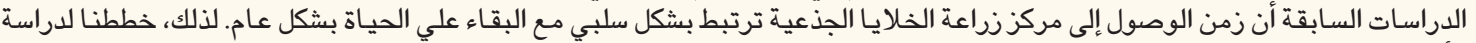

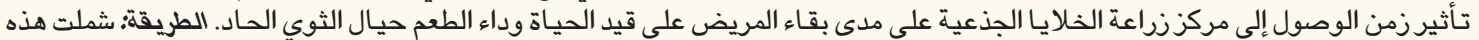

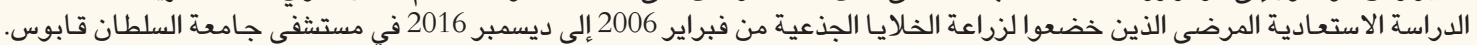

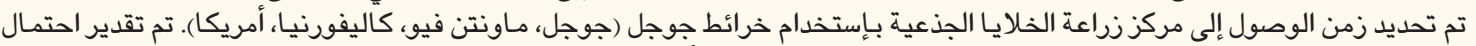

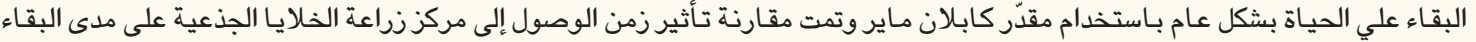

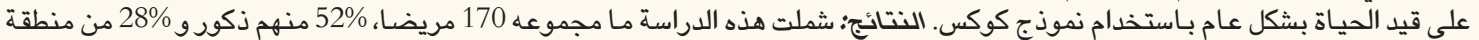

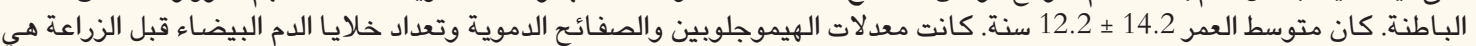

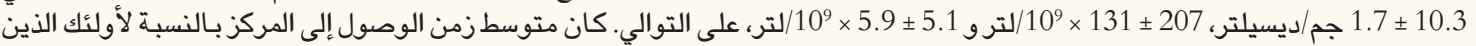

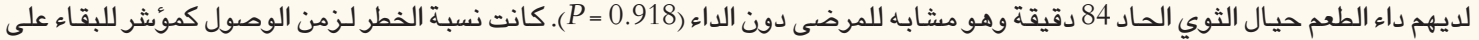

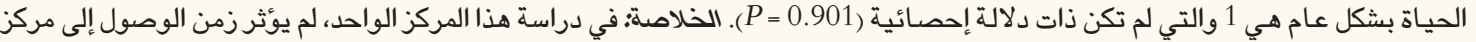

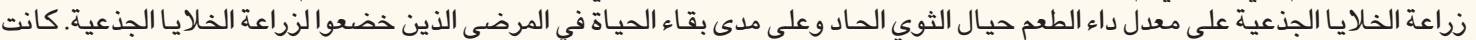

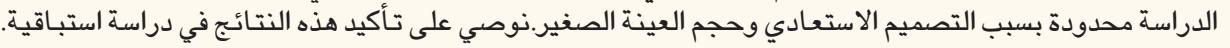

الكلمـات المفتاحية: زراعة الخلايا الجذعية المكونة للدم؛ داء الطعم حيال الثوي؛ تحليل البقاء على الحياة؛ السفر؛ عمان.

\section{ADVANCES IN KNOWLEDGE}

This study found that home-to-transplant centre distance had no impact on the survival in patients who receive haematopoietic stem cell transplantation or on their risk of acute graft-versus-host disease.

\section{Application to patient CARE}

Home-to-transplant centre distance should not impact the decision to proceed with haematopoietic stem cell transplantation (HSCT). Physicians should ensure adequate follow-up for patients post-HSCT regardless of their home-to-transplant centre distance.

$\mathrm{H}$ AEMATOPOIETIC STEM CELL TRANSPLANTation(HSCT) includes peripheral blood stem cell, umbilical cord stem cell and bone marrow transplantations. HSCT is used as a treatment modality to replace congenital or acquired life-threating abnormal bone marrow with normal haematopoietic stem cells to allow haematopoietic reconstitution after chemotherapy or chemoradiotherapy. ${ }^{1}$ A number of complications are associated with $\mathrm{HSCT}$, including infections, haemorrhage, graft failure, secondary malignancies and chronic and acute graft-versus-host disease (aGvHD). ${ }^{2}$ 
Home-to-transplant centre distance, estimated as driving time to the transplant centre (DTC), has been shown to affect a number of transplant outcomes, albeit not consistently. Abou-Nassar et al. found that overall survival (OS) after HSCT was $76 \%$ for patients who spent $\leq 40$ minutes driving time and $65 \%$ for patients with $\geq 160$ minutes driving time. ${ }^{3}$ Contrary to Abou-Nassar et al's study, Graupner et al. found no impact of DTC on post-transplant hospital length of stay, hospital readmission in the first 100 days and OS at day 100 and at one year. ${ }^{4}$

This study hypothesised that frequent early posttransplant follow-up of patients living far from the transplant centre may be difficult to adhere to and result in patients not attending as advised. This may impact early complications such as aGvHD and treatment related mortality. The impact of travelling distance has not been investigated on the outcomes of HSCT in Oman or in the region. Sultan Qaboos University Hospital (SQUH), Muscat, Oman is a tertiary care national institute in the capital city with over 600 beds and serves as the only allogeneic HSCT centre in Oman, treating patients from the entire country. Patients have to travel to the transplant centre for their follow-up, which could be up to twice per week early in the post-transplant period. This study aimed to determine the impact of DTC on OS and the probability of developing aGvHD for patients undergoing HSCT at SQUH.

\section{Methods}

This retrospective study was conducted between February 2006 and December 2016 and included all patients who received HSCT at the transplant centre of SQUH, regardless of indication. In addition, patients who had received HSCT outside the country and had arrived in Oman before day 100 of the HSCT date were included. Patients with missing data on OS or on aGvHD development were excluded. Furthermore, patients who received autologous HSCT as the follow-up in the early post-transplant period were also excluded as this is different than allogeneic HSCT and there is no risk of developing aGvHD. The decision of the preparative regimen was made using a consensus decision during a bone marrow transplantation board discussion.

Driving distance and time from the primary residence of patients to SQUH was determined using Google Maps, Version 3.32 (Google LLC., Mountain View, California, U.S.A) and was considered a continuous variable in the analysis of this study. Given that the DTC may change during the day as estimated by Google Maps (Google LLC.) due to differing traffic conditions, this variable was estimated during the same time of the day and only during working days. Clinical and laboratory information were obtained from the patient's electronic health records.

Continuous variables were described using means with standard deviations or medians if the variable was not normally distributed. Categorical variables were described using frequencies and percentages. The probability of OS was projected using the Kaplan-Meier estimator and the impact of DTC on OS was compared using a Cox proportional hazard model. DTC was compared between patients with and without aGvHD using a t-test. Collected data were analysed using Statistical Package for Social Science (SPSS), Version 22 (IBM Corp., Armonk, New York, USA). An alpha threshold of 0.05 was used to determine statistical significance.

This study was reviewed and approved by the Ethics Committee of the College of Medicine and Health Sciences at SQUH (MRCE \#1598).

\section{Results}

A total of 170 patients were included in this study, with slightly more males than females (52\% versus $48 \%$ ). Most patients were from the Al Batinah region (28\%) [Figure 1]. The mean age, haemoglobin, platelet, white blood cell count and absolute neutrophil counts at the time of transplant were $14.2 \pm 12.2$ years, $10.3 \pm 1.7 \mathrm{~g} / \mathrm{dL}$, $207 \pm 131 \times 10^{9} / \mathrm{L}, 5.1 \pm 5.9 \times 10^{9} / \mathrm{L}$ and $2.5 \pm 4.8 \times 10^{9} / \mathrm{L}$, respectively. Patients were transplanted for various indications, including acute leukaemia $(n=41)$, sickle cell disease ( $n=28)$, immunodeficiency $(n=22)$, thalassaemia major ( $\mathrm{n}=12)$, lymphoma $(\mathrm{n}=8)$, Fanconi's anaemia $(n=7)$, severe aplastic anaemia $(n=7)$, chronic myeloid leukaemia $(n=4)$, paroxysmal nocturnal haemoglobinuria $(\mathrm{n}=3)$ and various other indications $(\mathrm{n}=38)$. Transplanted stem cells were collected from peripheral stem cells for 120 patients, from bone marrow for 49 patients and from both sources for one patient. All patients received matched sibling donors. The prophylaxis for aGvHD was either

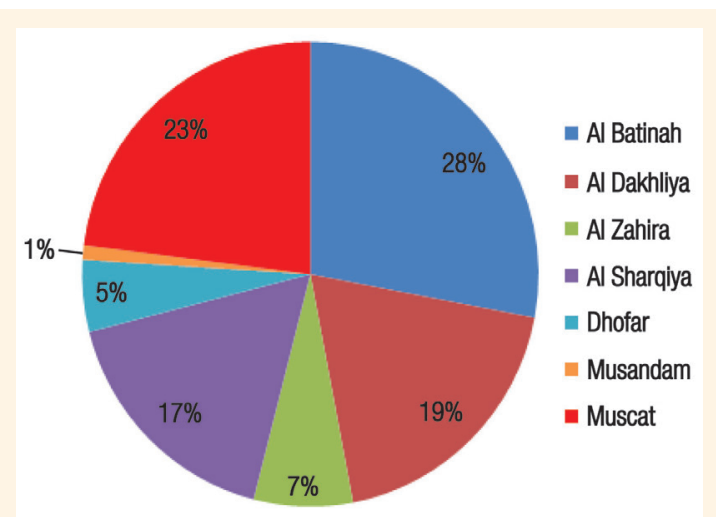

Figure 1: Distribution of all patients who received haematopoietic stem cell transplantation at the transplant centre of Sultan Qaboos University Hospital, Muscat, Oman according to the governorate of residence $(\mathrm{N}=170)$. 


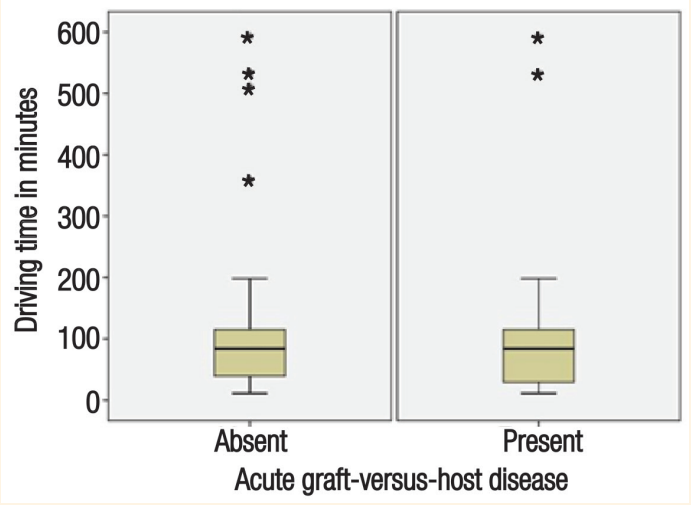

Figure 2: Driving time estimated using Google Maps (Google LLC., Mountain View, California, USA) of patients who received allogeneic haematopoietic stem cell transplantation with and without acute graft-versus-host disease from home to the transplant centre of Sultan Qaboos University Hospital, Muscat, Oman ( $\mathrm{N}=170)$.

cyclosporine or tacrolimus with a short course of methotrexate for all patients. The median driving distance and time were $137 \mathrm{~km}$ (range: 6.6-997 km) and 84 minutes (range: 11-594 minutes).

Figure 2 shows the boxplot distribution of the DTC variable in patients with and without aGvHD. Both distributions were similar and had a similar median DTC of 84 minutes with no statistically significant difference $(P=0.918)$. The mean of the DTC was $106 \pm 115$ minutes in patients without aGvHD and $110 \pm 137$ minutes in patients with aGvHD. The probability of OS for all patients at 2 and 5 years after HSCT were $89 \%$ and $80 \%$, respectively. Figure 3 illustrates the probability of OS over time in patients lower and higher than the median DTC. The impact of DTC on OS as assessed by a Cox proportional hazard model revealed a hazard ratio of 1.0 with no statistically significant impact of DTC on the OS $(P=0.901)$. Given the lack of impact in the univariable analysis of

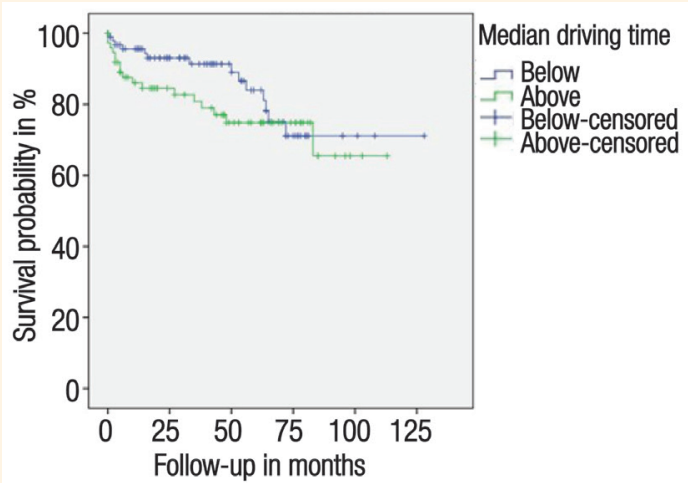

Figure 3: Overall survival of patients who received haematopoietic stem cell transplantation. The $y$-axis represents the Kaplan-Meier estimate of the overall survival probability according to the median driving time from home to the transplant centre at Sultan Qaboos University Hospital, Muscat, Oman $(\mathrm{N}=170)$.
DTC on both OS and aGvHD, multivariable analyses were not performed in the current study.

\section{Discussion}

A number of studies have addressed disparities in cancer survival rates based on geographic proximity to the treatment center. ${ }^{5,6}$ However, studies which examine the impact of home-to-transplant centre distances on the OS and risk of developing aGvHD in patients undergoing HSCT are scarce. ${ }^{3-6}$ To the best of the authors' knowledge, this is the first study to address this issue in Oman or in the region.

A retrospective cohort study from Nebraska assessed whether the primary area of residence was an independent risk factor for OS in patients undergoing autologous and allogeneic HSCT. ${ }^{4}$ A significant impact was found among patients undergoing autologous HSCT, but not with allogeneic HSCT. Those from rural areas were found to have a significantly lower survival rate (73\%) compared to those from urban areas $(78 \% ; P=0.04){ }^{4}$ However, the hazard ratio observed in this study provides evidence that patients from rural areas undergoing allogeneic HSCT are not at a greater risk of mortality than patients from urban areas. Associations found in other studies may be related to the type of health insurance coverage in urban versus rural areas. In Oman, all Omanis have governmental health insurance, which covers HSCT. This may have led to the lack of impact of DTC on OS in this study as all patients, regardless of their area of residence, had equal access to healthcare. The differences in the transport system of Nebraska and Oman may have led to some of the differing outcomes observed between the two studies. ${ }^{4}$ Given the previous literature, DTC was not expected to have an impact on autologous HSCT and the expected low mortality from this kind of transplantation lead to its exclusion from the current study. ${ }^{4}$

The gender ratio in the current study was approximately equal, which is representative of the ratio in the general Omani population. ${ }^{7}$ Most of the patients were from the Al Batinah and Muscat regions, which reflects the population distribution in Oman. No significant relationship was found between the area of residence and OS as assessed by the Cox regression analysis. Additionally, Omani patients receiving HSCT are generally younger than those reported in studies from Europe and North America. ${ }^{8}$ Therefore, the association between DTC and OS may not be as strong in younger patients when compared to older patients. Finally, many of the patients who received HSCT in the present study were transplanted for benign conditions that generally have a better prognosis than patients transplanted for malignant conditions. ${ }^{8}$ 
Furthermore, data suggests that an increase in DTC is not associated with the development of aGvHD $(P=0.918)$. Al-Khabori et al's study showed that the incidence of aGvHD in patients transplanted at SQUH was $32 \%$ while the incidence of chronic GvHD was $16 \%{ }^{8}$ Similar reasons as for the OS outcome could also be applicable for the aGvHD outcome. The development of aGvHD has been inconsistently shown to impact OS in international studies. ${ }^{3}$

There are a number of limitations in the present study. Since this was a retrospective study, there is a high chance of selection bias. Additionally, the small sample size most likely contributed to the low statistical power and therefore affects the ability to make definitive conclusions. The use of Google Maps (Google LLC.) online application to estimate driving time and distance from home to SQUH is another limitation as the application produced different driving times at different times of the day. This is likely due to the fact that Google Maps (Google LLC.) incorporates the current traffic conditions to estimate the driving time. Therefore, early morning estimates on working days were used for all patients to improve consistency and accuracy. Finally, this study spanned a large time period. The driving conditions and the supportive therapy may have changed during this period. The results of this study should be interpreted in light of these limitations. However, this study is the first to report the lack of impact of home-to-transplant centre distance and driving time on the OS of patients undergoing allogeneic HSCT in Oman as well as the risk of developing aGvHD.

\section{Conclusion}

This study suggests that driving distance is not associated with the mortality or the development of aGvHD. Larger and prospective studies should be conducted to more accurately assess the impact of DTC in the future in Oman and the region.

\section{CONFLICT OF INTEREST}

The authors declare no conflicts of interest.

\section{FUNDING}

No funding was received for this study.

\section{References}

1. Wilkinson IB, Raine T, Wiles K, Goodhart A, Hall C, O'Neill H. Oxford Handbook of Clinical Medicine. 10th ed. Oxford, UK: Oxford University Press, 2017. P. 352-62.

2. Hoffbrand AV, Moss PAH. Essential Haematology. 6th ed. New Jersey, USA: Wiley-Blackwell, 2011. P.251-61.

3. Abou-Nassar KE, Kim HT, Blossom J, Ho VT, Soiffer RJ, Cutler CS, et al. The impact of geographic proximity to transplant center on outcomes after allogeneic hematopoietic stem cell transplantation. Biol Blood Marrow Transplant 2012; 18:708-15. https://doi.org/10.1016/j.bbmt.2011.08.022.

4. Graupner S, Spellman A, Jacobson S, Bryan L, Sportes C, Pantin J, et al. Impact of socio-economic disparities and distance to bone marrow transplant center on transplantation outcomes. Biol Blood Marrow Transplant 2016; 22:S187. https://doi.org/10.1016/j.bb mt.2015.11.567.

5. Rao K, Darrington DL, Schumacher JJ, Devetten M, Vose JM, Loberiza FR Jr. Disparity in survival outcome after hematopoietic stem cell transplantation for hematologic malignancies according to area of primary residence. Biol Blood Marrow Transplant 2007; 13:1508-14. https://doi.org/10.1016/j.bbmt.2007.09.006.

6. Paulson K, Lambert P, Bredeson C, Demers A, Nowatzki J, Richardson E, et al. Does location matter? Rural vs urban outcomes after blood and marrow transplantation in a populationbased Canadian cohort. Bone Marrow Transplant 2010; 45:1167-73. https://doi.org/10.1038/bmt.2009.332.

7. Worldometers. Oman Population. From: www.worldometers. info/world-population/oman-population/ Accessed: Dec 2018.

8. Al-Khabori M, Al-Huneini M. Hematopoietic stem cell transplantation in the Sultanate of Oman. Hematol Oncol Stem Cell Ther 2017; 10:305-7. https://doi.org/10.1016/j.hemonc.2017.05.024. 